Short communication

\title{
Multiplexed electrokinetic sensor for detection and therapy monitoring of extracellular vesicles from liquid biopsies of non-small-cell lung cancer patients
}

\author{
Sara Cavallaro ${ }^{\mathrm{a},{ }^{* * *}}$, Petra Hååg ${ }^{\mathrm{b}}$, Siddharth S. Sahu ${ }^{\mathrm{c}}$, Lorenca Berisha ${ }^{\mathrm{d}}$, Vitaliy O. Kaminskyy ${ }^{\mathrm{b}}$,

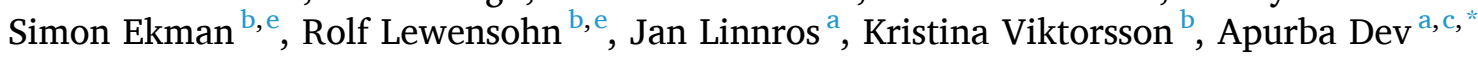 \\ ${ }^{a}$ Department of Applied Physics, School of Engineering Sciences, KTH Royal Institute of Technology, 10691, Stockholm, Sweden \\ ${ }^{\mathrm{b}}$ Department of Oncology/Pathology, Karolinska Institutet, 17164, Stockholm, Sweden \\ c Department of Electrical Engineering, The Ångström Laboratory, Uppsala University, 75121, Uppsala, Sweden \\ d Politecnico di Torino, 10129, Turin, Italy \\ e Theme Cancer, Patient Area Head and Neck, Lung, and Skin, Karolinska University Hospital, 17164, Solna, Sweden
}

\section{A R T I C L E I N F O}

\section{Keywords:}

Electrokinetic sensor

Multiplexing

Extracellular vesicles

Non-small-cell lung cancer

Liquid biopsy

Treatment monitoring

\begin{abstract}
A B S T R A T
Liquid biopsies based on extracellular vesicles (EVs) represent a promising tool for treatment monitoring of tumors, including non-small-cell lung cancers (NSCLC). In this study, we report on a multiplexed electrokinetic sensor for surface protein profiling of EVs from clinical samples. The method detects the difference in the streaming current generated by EV binding to the surface of a functionalized microcapillary, thereby estimating the expression level of a marker. Using multiple microchannels functionalized with different antibodies in a parallel fluidic connection, we first demonstrate the capacity for simultaneous detection of multiple surface markers in small EVs (sEVs) from NSCLC cells. To investigate the prospects of liquid biopsies based on EVs, we then apply the method to profile sEVs isolated from the pleural effusion (PE) fluids of five NSCLC patients with different genomic alterations (ALK, KRAS or EGFR) and applied treatments (chemotherapy, EGFR- or ALKtyrosine kinase inhibitors). The vesicles were targeted against CD9, as well as EGFR and PD-L1, two treatment targets in NSCLC. The electrokinetic signals show detection of these markers on sEVs, highlighting distinct interpatient differences, e.g., increased EGFR levels in sEVs from a patient with EGFR mutation as compared to an $A L K$-fusion one. The sensors also detect differences in PD-L1 expressions. The analysis of sEVs from a patient prior and post ALK-TKI crizotinib treatment reveals significant increases in the expressions of some markers (EGFR and PD-L1). These results hold promise for the application of the method for tumor treatment monitoring based on sEVs from patient liquid biopsies.
\end{abstract}

\section{Introduction}

Despite decades of research and development, cancer remains a major challenge for the global healthcare, leading to the death of millions of people every year (Sung et al., 2021). While the understanding of tumor biology as well as the methods of clinical interventions have seen major improvements, particularly regarding targeted therapy approaches, the progress on diagnostic technologies still needs to be improved. This has also been due to the reliance on tissue biopsy as a source of tumor material (Vaidyanathan et al., 2019). For a long time, tumor tissue biopsies have been the gold standard of cancer diagnostics and subtyping, yet they have limitations in treatment monitoring as they only provide a restricted information of the patient tumor burden, in addition to being invasive and not easily accessible in several cases (Vaidyanathan et al., 2019). Therefore, there has been an exponentially increasing interest in non-invasive sampling of body fluids, known as liquid biopsies, which offer a less invasive way for treatment monitoring (De Mattos-Arruda and Siravegna, 2021; Rolfo et al., 2018; Santarpia et al., 2018). Furthermore, liquid biopsies also give access to a plethora of ultrasensitive biosensing technologies, which have improved diagnostic approaches to many pathological conditions, including cancer (Vaidyanathan et al., 2019).

\footnotetext{
* Corresponding author. Department of Electrical Engineering, The Ångström Laboratory, Uppsala University, 75121, Uppsala, Sweden.

$* *$ Corresponding author.

E-mail addresses: saracav@kth.se (S. Cavallaro), apurba.dev@angstrom.uu.se, apurba.dev@angstrom.uu.se (A. Dev).
} 
The prospect of extracellular vesicles (EVs) as a source of tumor biomarkers in liquid biopsies has been highlighted in a large number of research articles (Li et al., 2021; Santarpia et al., 2018; Xu et al., 2018), as these vesicles carry proteins and mRNA/miRNA reflecting their cells of origin. Lung cancer (LC), one of the malignancies which causes most cancer related deaths (Sung et al., 2021) and where re-biopsy is challenging, has obviously been a subject of interest for EV-based diagnostics (Hoshino et al., 2020; Pasini and Ulivi, 2020; Sandfeld-Paulsen et al., 2016; Wan et al., 2018; Zhong et al., 2020). In particular, non-small-cell lung cancer (NSCLC), the major subtype of LC accounting for $\sim 80 \%$ of all LC (Planchard et al., 2018) and which has a 5-year survival rate of only $\sim 20 \%$ (according to Cancer.org), has drawn particular interest, both from the perspective of early stage detection as well as for monitoring of therapy responses (Pasini and Ulivi, 2020). Diagnostic biomarkers for NSCLC have been identified in both EV-protein profiles (An et al., 2019) and RNA content (Tao et al., 2020), thereby opening the scope for developing biosensing methodologies targeting either of the two biomarker types. During the past years, different biosensing techniques, e.g., electrochemical, surface plasmon resonance (SPR) or nanoshearing, have been developed for detection and protein profiling of EVs (Jeong et al., 2016; Sina et al., 2016; Vaidyanathan et al., 2014). In the context of NSCLC, while different methodologies, e.g., lateral flow aptamer assay (Yu et al., 2020) or SPR imaging (SPRi) array (Fan et al., 2020), have recently demonstrated the capacity of surface protein profiling of NSCLC cell derived EVs, the scope for clinical sample analysis has been mainly investigated by traditional proteomic analyses and sequencing-based methods (Hoshino et al., 2020; Hydbring et al., 2018; Krug et al., 2018; Sandfeld-Paulsen et al., 2016).

In comparison, the detection method employing the electrokinetic principles of streaming current/potential offers a simpler alternative for EV analysis. The method allows label-free detection of target molecules/ bioparticles within a microfluidic channel by exploiting hydrodynamic and electrostatic interactions at the solid-liquid interface (Adamczyk et al., 2010). Moreover, the technique has a simple design and good sensitivity. While in the past, the principle has been applied by various research groups to detect a variety of biomarkers (Dev et al., 2016; Koch et al., 1999; Martins et al., 2011), we were the first to demonstrate the possibility to use streaming current method for detection of EVs (Cavallaro et al., 2019). In particular, in our previous study, we demonstrated the capability of this method for detection and profiling of EVs from cell lines using a single-channel measurement configuration (Cavallaro et al., 2019). The technique was able to discriminate changes in epidermal growth factor receptor (EGFR) expression in EVs isolated from a NSCLC cell line, with a sensitivity of $10 \%$ of the parental cell expression (Cavallaro et al., 2019). Besides, only a small amount of sample $(\sim 12 \mu \mathrm{L})$ was necessary for such measurement. Since mutated EGFR is often targeted in NSCLC using tyrosine kinase inhibitors (TKIs) (Guo et al., 2020; König et al., 2021; Suda et al., 2017), the method holds a substantial promise for further validation in clinical samples. However, a major challenge in analyzing EVs isolated from liquid biopsies of patients is the heterogeneity of the vesicles, the complex matrix of plasma, which also contains proteins that might get co-isolated with EVs, and also low sample volume (Ferguson and Weissleder, 2020). This means that, for such clinical applications, a biosensing method needs to have the capacity to measure multiple targets to simultaneously assess the changes in multiple EV surface markers among different patients and/or treatment lines. This has to be performed with a high specificity and reproducibility, however with a small amount of sample. To the best of our knowledge, such capacity has not been demonstrated using this sensing method.

In this article, we first validated the possibility to use a multiplexed electrokinetic platform based on streaming current (Is) for the detection of EVs. For this purpose, we used vesicles isolated from cell culture media of NSCLC cells and characterized the platform for the simultaneous measurements of four microcapillary sensors. Following validation, for the first time we applied the multiplexed platform for detection and profiling of EVs from clinical samples. In particular, the vesicles were isolated from malignant pleural effusion (PE) fluids of five NSCLC patients with different genomic alterations and during different stages of their treatment courses, and two non-cancerous patients. PE refers to the accumulation of fluid in the space between the lungs and the chest walls in both malignant and non-malignant conditions (Baburaj et al., 2020; Stiller et al., 2021). For advanced LC patients, PE fluid accumulation is seen in about $10-15 \%$ of all cases with a predominance of adenocarcinomas due to their growth pattern in the lung (Baburaj et al., 2020). As malignant PE fluid is enriched in tumor cells, it might also contain tumor cell derived EVs and can be collected in a minimally invasive way, it represents a suitable source for liquid biopsies based on EVs. For the analysis, we targeted CD9 tetraspanin and two biomarkers of interest in NSCLC, namely EGFR and PD-L1, expressed on the EV surfaces. The results demonstrated successful detection of EVs from PE samples, highlighting interpatient differences in the expression of the analyzed markers. Finally, aiming to examine the possibility to use the multiplexed electrokinetic platform for treatment monitoring, we analyzed the EVs from an ALK-positive NSCLC patient at two time points during the treatment course. Particularly, we compared the expression levels of CD9, EGFR, PD-L1 and HER2 on EVs from PEs collected prior and post ALK-TKI crizotinib treatment. The data suggested increased levels of some of the analyzed markers, e.g., EGFR and PD-L1, while showing stable expressions of other ones, e.g., CD9.

\section{Materials and Methods}

The methods and materials used for the development and experimental validation of the detection platform along with the isolation technique for sEVs and their characterizations are presented in the following sections.

\subsection{Reagents}

High purity deionized water (DIW) with a resistivity of $18 \mathrm{M} \Omega \mathrm{cm}$ was used throughout all the experiments. Phosphate-buffered saline (PBS, P4417) in tablets was purchased from Sigma-Aldrich. Cetuximab antibody (Erbitux infusion solution, L01XC06, $5 \mathrm{mg} / \mathrm{mL}$, Merck Serono) was used to target EGFR. Anti-CD9 (MEM-61), anti-CD63 (mab5048), anti-HER2 (HRB2/258), anti-IGF1R (1H7), anti-PD-L1 (MAB1561) and isotype control (MAB002) antibodies were purchased from Bio-Techne. If not stated otherwise, all the other chemicals were purchased from Sigma-Aldrich Sweden AB.

\section{2. $s E V$ collection and isolation}

The EVs investigated in this study were isolated in order to obtain small EVs (sEVs, $\sim 30-300 \mathrm{~nm}$ ). The sEVs used to characterize the multiplexed platform were collected from the cell culture media of a NSCLC cell line H1975 (ATCC® CRL-5908 ${ }^{\mathrm{TM}}$, LGC Standards, Teddington Middlesex, United Kingdom) with two mutations in EGFR (exon 20, T790M and exon 21, L858R) (Pao et al., 2005). The sEVs from patients were isolated from the malignant PE fluids of five NSCLC patients, here named as PE002, PE009, PE010, PE011 and PE017, and from two non-cancerous patients, here named as RP078 and RP025. The patient NSCLC tumors contained genomic alterations in EML4-ALK variant 3 (a/b) (PE002 and PE017), KRAS exon 2, codon 12/13 (PE009), and EGFR exon 21, L858R (PE010 and PE011), respectively. These samples were collected at Karolinska University Hospital, Stockholm, Sweden. The study was approved by the Ethics Review Authority in Sweden (https://etikprovningsmyndigheten.se), region Stockholm (EPN No. Dnr. 2016/2585-32/1, approval date March 8, 2017), with patient informed consent to study participation. The study was also granted biobank approval with respect to tumor material into biobank and transfer for analyses at Uppsala University by Material Transfer Agreement. Both the NSCLC cell line and PE fluid EV samples were isolated by 
size exclusion chromatography (SEC) using qEVoriginal Columns (Izon Science, Oxford, United Kingdom). In particular, the isolation of the sEVs from the cell culture media of NSCLC cells followed the protocol reported in our earlier studies (Cavallaro et al, 2019, 2021). For the PE fluid sEVs, we followed the procedure described in another earlier work (Stiller et al., 2021).

\section{3. sEV characterization}

The sEVs from cell culture media of NSCLC cells were characterized by nanoparticle tracking analysis (NTA) for their size and particle count estimations. As presented in Fig. S1, the vesicles showed the expected size profile. In our earlier study, the same sEVs were further characterized by scanning electron microscopy (SEM), showing the expected vesicle sizes and morphologies (Cavallaro et al., 2021). Western Blot (WB) analysis was performed to verify the presence of sEV markers, e.g., CD9, EGFR and TSG101, and the absence of cellular contamination by calnexin, as previously described (Cavallaro et al., 2019). The expression of HER2 and IGF-1R in sEVs was examined by proximity extension assay (PEA) due to the higher sensitivity of the method as compared to WB. The analysis was performed on the multiplex Immune Oncology and Oncology II ${ }^{\circledR}$ panel (Olink Proteomics AB, Uppsala, Sweden) carried out at the Clinical Biomarker Facility, Science for Life Laboratory, Uppsala University, Uppsala, Sweden. The PEA assay consists of antibody pairs against proteins linked to different tumor or immune signalling processes (https://www.olink.com/products) and also harbor internal positive and negative controls of the assay. For analyses, sEVs were lysed in 5x RIPA buffer to a final concentration of $1 \mathrm{x}$ RIPA $(50 \mathrm{mM}$

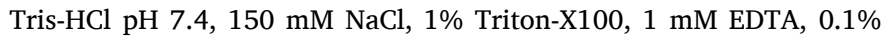
SDS) from which $1 \mu \mathrm{L}$ was applied in the assay. Data processing was carried out on the Olink Wizard for GenEx software, and the normalized protein expression (NPX) values were used in subsequent analyses. The fold levels relative to each marker's limit of detection (LOD) value are given for the samples. The size profiles and concentrations of the sEVs from the NSCLC and non-cancerous patient PE fluids were also characterized by NTA. For their biomarker characterization, WB (for EGFR, $\mathrm{CD}$, no calnexin) and proximity extension assay (PEA, for PD-L1) were used. The results of the analyses are reported in an earlier study ((Stiller et al., 2021), NTA profiles and WB) and in Supplementary Information (Fig. S2, NTA, WB and PEA). The details on NTA and WB analyses are provided in our earlier studies (Cavallaro et al., 2021; Stiller et al., 2021).

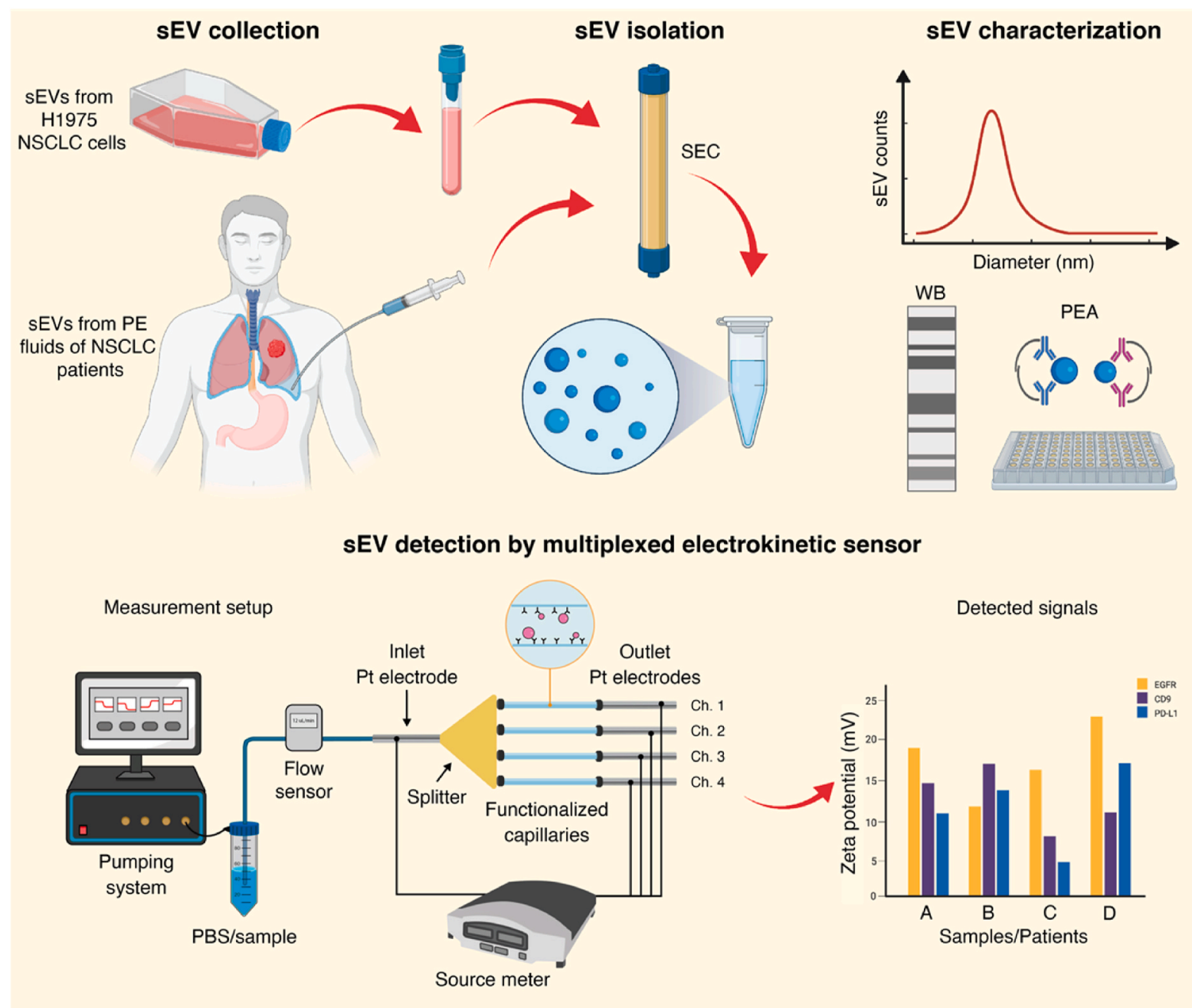

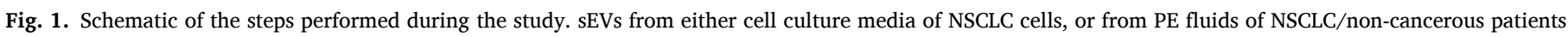

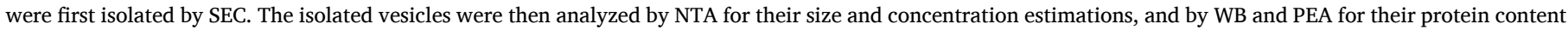

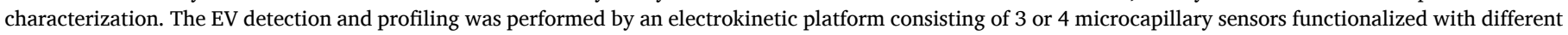

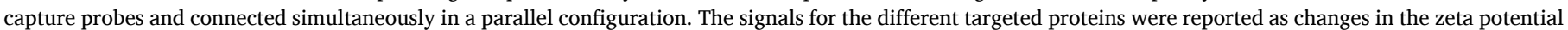
between second and initial PBS baselines and compared among different samples. This figure was created using BioRender.com. 


\subsection{Multiplexed electrokinetic platform}

Fig. 1 shows a schematic of the measurement process, from the sEV isolation and characterization to the electrokinetic detection and profiling. Following isolation and characterization, electrokinetic experiments were performed in a multiplexed platform consisting of a commercial pumping system (Elveflow, OB1), a flow sensor (Elveflow, MFS3), a fluidic system including the microcapillary sensors, and a measurement unit (Keithley source meter and PC). The multiplexed nature of the setup allowed the simultaneous measurement of multiple markers on the EV surface, while using a low amount of sample. These aspects are essential for the use of EVs from liquid biopsies as a biomarker for disease monitoring. As compared to our previous study (Cavallaro et al., 2019), which consisted of only one channel (as shown in Fig. S3), the fluidic system of the presented multiplexed platform comprised microfluidic tubing to transport the buffer/EVs from the sample vials to the sensors, a multiport connector for equal sample splitting and multiple microcapillary sensors (inner diameters of $25 \mu \mathrm{m}$ and lengths of $\sim 4.7 \mathrm{~cm}$ ). Moreover, here we chose to connect the multiple sensors in a parallel configuration instead of a series one, shown in Fig. S3. For the purpose, a hollow Pt tube was used as a common electrode for all sensors at the inlet (before multiport connector), while separate Pt electrodes were connected to the outlet of each microcapillary. For each sensor, the streaming current between the Pt electrode at the inlet and the one at the outlet was independently measured.

The microcapillary sensors were functionalized following the main steps of our previously reported protocol (Cavallaro et al., 2019). Briefly, the capillaries were cleaned using a solution of DIW, hydrogen peroxide, ammonium hydroxide in $5: 1: 1$ ratio at $88{ }^{\circ} \mathrm{C}$ for $10 \mathrm{~min}$. Thereafter, following (3-aminopropyl)triethoxysilane (APTES) activation and glutaraldehyde (GA), the antibodies targeting the different EV surface proteins were immobilized to the inner microcapillary surfaces for $2 \mathrm{~h}$, at a concentration of $50 \mu \mathrm{g} / \mathrm{mL}$ in $1 \mathrm{x}$ PBS. Tris-Ethanolamine (30 $\mathrm{min})$ and casein $(2 \mathrm{~h})$ were then used to deactivate the surfaces, thus reducing non-specific binding (NSB) of the vesicles (Cavallaro et al., 2019). However, in this work two main modifications were introduced as compared to our previous functionalization strategy. First, the choice of the targeting antibodies was optimized by selecting those with a more positive charge as compared to the previous ones (Cavallaro et al., 2019). This increased the contrast between the surface charge of the sensors and that of the EVs, leading to higher detected signals. Moreover, here we introduced a different type of control sensors, based on isotype control antibodies instead of functionalized capillaries without capture probes (Cavallaro et al., 2019). These control antibodies were immobilized at the same concentration as the targeting ones $(50 \mu \mathrm{g} / \mathrm{mL}$ in $1 \mathrm{x}$ PBS), representing a better strategy to test the EV NSB, especially when analyzing clinical samples. Prior to measurements, all microfluidic tubing were also treated with casein for $2 \mathrm{~h}$ to minimize NSB of the vesicles.

For detection, we analyzed the streaming current (Is) induced by a pressure driven flow of a $0.1 \times$ PBS buffer (conductivity $=0.17 \mathrm{~S} / \mathrm{m}$ ) and measured the current changes upon sEV binding to the functionalized microcapillary surfaces, as reported in our previous study (Cavallaro et al., 2019). In brief, for each channel, we measured the Is for a continuous periodic (30 s pulse duration) rectangular pulse of $1.5 \mathrm{bar}$ pulse height (1.5 bar and 3.0 bar applied pressures), which was used to drive the PBS solution through the microcapillaries. The current difference $(\Delta I s)$ for the applied pressures was recorded by the source meter and converted in apparent zeta potential $\left(\zeta^{*}\right)$, according to the equation:

$\zeta^{*}=\frac{\Delta I_{s}}{\Delta P} \frac{\eta}{\varepsilon \varepsilon_{0}} \frac{L}{A}$

where $\Delta \mathrm{Is} / \Delta \mathrm{P}$ is the change in Is with pressure, $\eta$ the viscosity at $\sim 23{ }^{\circ} \mathrm{C}$ $(\sim 0.9 \mathrm{cP}), \varepsilon \varepsilon_{0}$ the permittivity, and L/A the length and cross-section of the capillaries, respectively. The collected data were analyzed by a
A

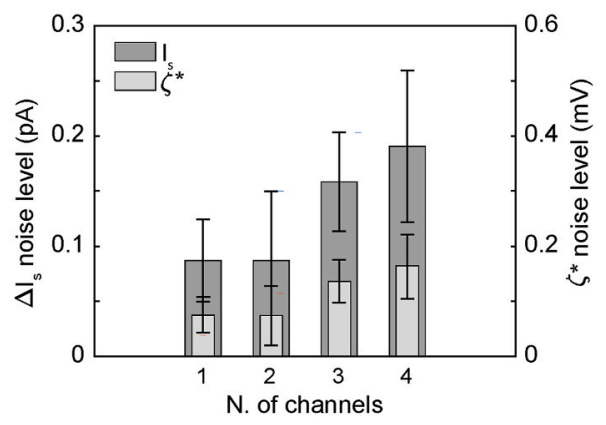

C

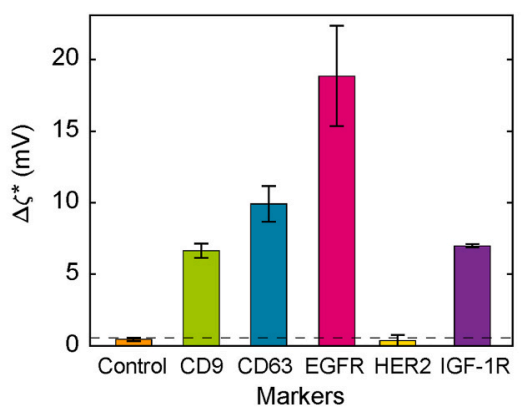

B

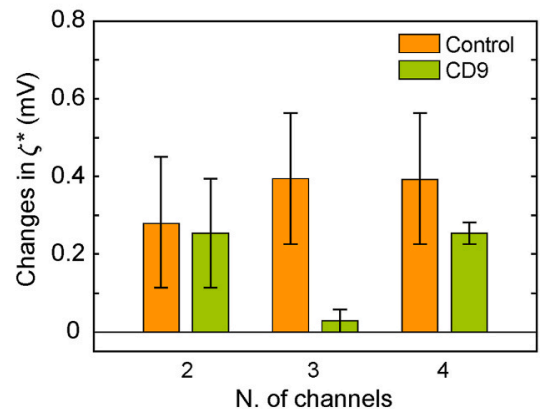

$\mathrm{E}$

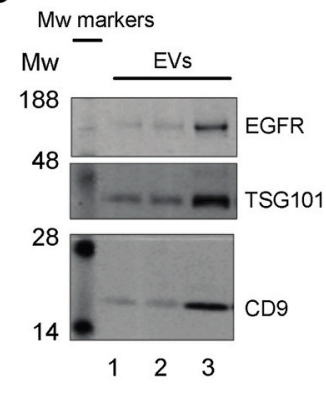

Fig. 2. Multiplexed electrokinetic platform characterization and validation with sEVs from conditioned cell culture media of NSCLC H1975 cells. (A) Noise levels in the $\Delta \mathrm{I}_{\mathrm{s}}$ (left y-axis) and corresponding $\zeta^{*}$ (right $y$-axis) for single-to four-channel configuration measurements, calculated for 5 different microcapillaries. (B) $\zeta *$ changes in the initial baselines (in absolute values) when measuring two to four simultaneous channels as compared to the corresponding single-channel measurements. An EGFRfunctionalized and a second control capillary were connected as 3rd and 4th channels (not shown in the plot). Measurements performed in duplicate. (C) Sensor responses for the different capture probes CD9, CD63, EGFR, HER2 and IGF-1R measured by the multiplexed platform. sEVs incubated in the microcapillary sensors at a concentration of $3.5 \times 10^{9}$ particles $/ \mathrm{mL}$. Measurements performed in duplicate. The dashed line depicts the MDS of the platform in its four-channel configuration measurement $(\sim 0.5 \mathrm{mV})$. (D) Western blot analysis of sEVs, showing the presence of EV markers, e.g., CD9, TSG101, the tumor marker EGFR. The blot was also probed with the ER-protein calnexin which did not show any band (data not shown). The different lanes correspond to the different amounts of EVs that were analyzed. In particular, lane 1: $7.1 \times 10^{7} \mathrm{EVs}$, lane 2:

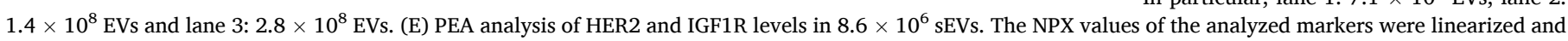
fold increase relative to limit of detection (LOD) values for each marker are shown. 
custom-designed MATLAB software. All experiments were performed at room temperature $\left(\sim 23^{\circ} \mathrm{C}\right)$, for the sake of simplicity, and in $\mathrm{PBS}$ at $\mathrm{pH}$ 7.4, to mimic physiological conditions. However, the PBS buffer was diluted to $0.1 \times$ to decrease the concentration of counterions, thereby increasing the magnitude of the measured $\zeta *$ (Kirby and Hasselbrink, 2004). In our previous work, we showed the integrity of the EVs at these conditions for the duration of all the experiments (Cavallaro et al., 2019). Moreover, we followed an end-point detection approach, as described earlier (Cavallaro et al., 2019). Specifically, two baselines were recorded, one prior to and one post SEV incubation in the microcapillaries, and the signals were calculated as the difference in the $\zeta^{*}$ between these two baselines $\left(\Delta \zeta^{*}\right)$. For all measurements, the EVs were incubated at a concentration of $3.5 \times 10^{9}$ particles $/ \mathrm{mL}$. This concentration was selected based on EV concentration in the extracted sample, the LOD of the platform (Cavallaro et al., 2019), the possibility to scan multiple markers from a small amount of sample and also to compare the results with our previous work (Cavallaro et al., 2019). Moreover, for all the measurements we ensured that an equal flow rate was maintained across the different channels and over time, as shown in Fig. S4. Following each measurement, the setup including microfluidic tubing, flow sensor and electrodes, was cleaned using i) a solution of $5 \% \mathrm{w} / \mathrm{v}$ sodium dodecyl sulfate (SDS) and $0.5 \mathrm{M}$ acetic acid, ii) acetone, iii) isopropanol and iv) air flow. This cleaning procedure led to reproducible baselines over different measurements (for the same antibody functionalization) and the absence of significant current drift over time (Fig. S5). The measurement results were analyzed in MATLAB and the data from the replicates were plotted as mean value $+/$ - standard deviation (SD).

\section{Results}

\subsection{Validation of multiplexed platform with EVs from NSCLC H1975} cells

To set up and characterize the multiplexed electrokinetic platform using a parallel sensor configuration, we first tested the effects of multichannel connection on the measurement parameters. In particular, we compared the noise in current measurements and the changes in the baseline values upon connection of 1-4 simultaneous microcapillary sensors. We selected a maximum of 4 parallel channels for the proof of principle study, as this could be easily setup by using commercially available manifold connectors. Fig. 2A shows the noise in the $\Delta \mathrm{I}_{\mathrm{s}}$, calculated as the standard deviation of a blank (peak values), for microcapillaries in different channel configurations. For easier comparison with the following data reported in the study, we also included the corresponding noise levels in $\zeta^{*}$ (Fig. 2A, inset bar plots). For the noise analysis, we considered microcapillaries functionalized with the different capture probes reported in Materials and Methods (CD9, cetuximab, HER2, CD63, IGF-1R). As visible in Fig. 2A, the noise level in the two-channel configuration remained the same as in the singlechannel measurement $(\sim 0.1 \mathrm{pA} / 0.07 \mathrm{mV})$, while showing a 1.5 - and a 2 -fold increase in presence of 3 and 4 simultaneous channels, respectively. The noise data from the different channel configurations was then used to calculate the minimum detectable signal (MDS) for each particular configuration. Specifically, we defined MDS as three times the current/zeta potential noise. Further, to test if there was any crossinterference between the different channels, we measured the changes of the initial baseline values ( $\zeta^{*}$ prior to EV incubation) upon connection of 2,3 and 4 simultaneous capillaries, with respect to their corresponding single-channel baselines. Fig. 2B shows these results for a CD9antibody functionalized sensor and a control one. As a reference, we set the corresponding initial single-channel baselines as $\zeta^{*}=0 \mathrm{mV}$ and reported the changes in absolute $\zeta^{*}$ values.

As presented, both sensors showed negligible baseline variations, in the order of the MDS of the corresponding numbers of channels. This suggested the absence of cross-interference, indicating that each capillary sensor could be measured independently of the others. However, we noticed a slightly unequal distribution of the buffer among the different microcapillaries when connecting 4 channels simultaneously $(\sim 13 \%$ variation in $\Delta$ flowrates). The flow was instead distributed more uniformly ( $<7 \%$ variation) when connecting 2 or 3 channels in parallel, as shown in Fig. S4.

Following initial characterization, we tested our four-channel multiplexed platform with sEVs isolated from conditioned cell culture media of a NSCLC cell line H1975. For the analysis, we targeted the ubiquitous EV tetraspanins CD9 and CD63, and some biomarkers of interest in NSCLC therapy, e.g., EGFR, HER2 and IGF-1R. These tumor cell surface receptors are known to be oncogenic drivers of NSCLC. EGFR is also a precision medicine target for EGFR-mutated NSCLC, while HER2 and IGF-1R are both reported to be bypass drivers in EGFR-TKI refractoriness (König et al., 2021; Lai-Kwon et al., 2021). As presented in Fig. 2C, all the functionalized sensors showed a positive response as compared to control capillaries (with isotype control antibodies), except for HER2. We could not detect this protein using the selected antibody and sEV concentration $\left(3.5 \times 10^{9}\right.$ particles/mL). For comparison, we also analyzed the same surface markers using WB and PEA. The results are reported in Fig. 2D-E and confirmed the expression of these proteins in sEVs from NSCLC H1975 cells.

\subsection{Detection of sEVs from malignant PE fluids of NSCLC patients}

Following characterization and validation with sEVs from NSCLC cells, we tested whether our multiplexed electrokinetic platform could detect EVs from patient liquid biopsy samples and discriminate in their surface protein expression levels. Fig. 3A summarizes details on the tumor types, stages at diagnosis and treatment courses at sample collection for the analyzed NSCLC patients.

In details, we analyzed the sEVs isolated from the PE fluid samples of five adenocarcinoma NSCLC patients and of two patients without malignant tumor cells, hence considered non-cancerous or healthy. The adenocarcinoma patients had different genomic alterations, namely EML4-ALK variant 3 (a/b) (PE002 and PE017), KRAS exon 2, codon 12/ 13 (PE009), and EGFR exon 21, L858R (PE010 and PE011), respectively. PE002 and PE017 patients were on ALK-TKI crizotinib when PE samplings were obtained and responded to this treatment line. PE009 patient was on standard chemotherapy at the time of sampling with stable disease. PE011 and PE010 patients were given EGFR-TKI erlotinib, responding and showing resistance to this treatment, respectively. For electrokinetic detection, we investigated the transmembrane proteins CD9, EGFR and PD-L1 and compared their levels in the sEVs isolated from the five adenocarcinoma NSCLC PE samples. We included PD-L1 in the analysis as it has been demonstrated that the tumor expression of this marker may impede the immune system to attack the tumor, thereby offering a way to monitor therapy (Abdel Karim and Kelly, 2019; D. H. Kim et al., 2019). PD-L1 is also reported to be expressed in EVs (Wang et al., 2021) and EGFR-TKIs treatment of NSCLC patients has been demonstrated to alter PD-L1 expression and other signalling components in the immune landscape (Kang et al., 2021; Liu et al., 2021). For this investigation, we used the platform in a three-channel configuration, in order to reduce the inhomogeneous flow distribution among the capillaries that was detected when connecting four channels simultaneously. For comparison, we analyzed the same sEV batches for CD9, EGFR and PD-L1 using WB and PEA. These results are presented in an earlier work (Stiller et al., 2021) and in Fig. S2. The bar plots in Fig. 3B show the sensor responses (upon control subtractions) for the five NSCLC patient samples and surface proteins analyzed. Control measurements (capillaries with isotype control antibodies) showed lower responses (Fig. S6) than the respective signals. However, in some cases, increased NSB were detected ( $\sim 5.5$-fold for PE009, $\sim 7.5$-fold for PE017 and $\sim 20$-fold for PE010, Fig. S6) as compared to the controls obtained with sEVs from the NSCLC cell line $(\sim 0.5 \mathrm{mV}$, Fig. $2 \mathrm{C})$. As presented in Fig. 3B, the electrokinetic data revealed positive detection of both CD9 and EGFR for the 
A

\begin{tabular}{|lllll|}
\hline Patient & Tumor histology & Genomic alteration & Tumor stage at diagnosis & Treatment at PE fluid collection \\
\hline PE002 & Adeno & EML4-ALK variant 3 $(\mathrm{a} / \mathrm{b})$ & T2aNOM1a & ALK-TKI Crizotinib, responsive \\
PE002-2 & Adeno & EML4-ALK variant 3 $(\mathrm{a} / \mathrm{b})$ & T2aNOM1a & ALK-TKI Crizotinib, refractory \\
PE009 & Adeno & KRAS exon 2, codon 12/13 & T4N3M1b & Carboplatin/Navelbine, stable \\
PE010 & Adeno & EGFR exon 21, L858R & T4N2M1b & EGFR-TKI Erlotinib, refractory \\
PE011 & Adeno & EGFR exon 21, L858R & T4N2M1a & EGFR-TKI Erlotinib, responsive \\
PE017 & Adeno & EML4-ALK variant 3 $(\mathrm{a} / \mathrm{b})$ & T4N2M1c & ALK-TKI Crizotinib, responsive \\
RP025 & Non-cancerous & NA & NA & NA \\
RP078 & Non-cancerous & NA & NA & NA \\
\hline
\end{tabular}

B

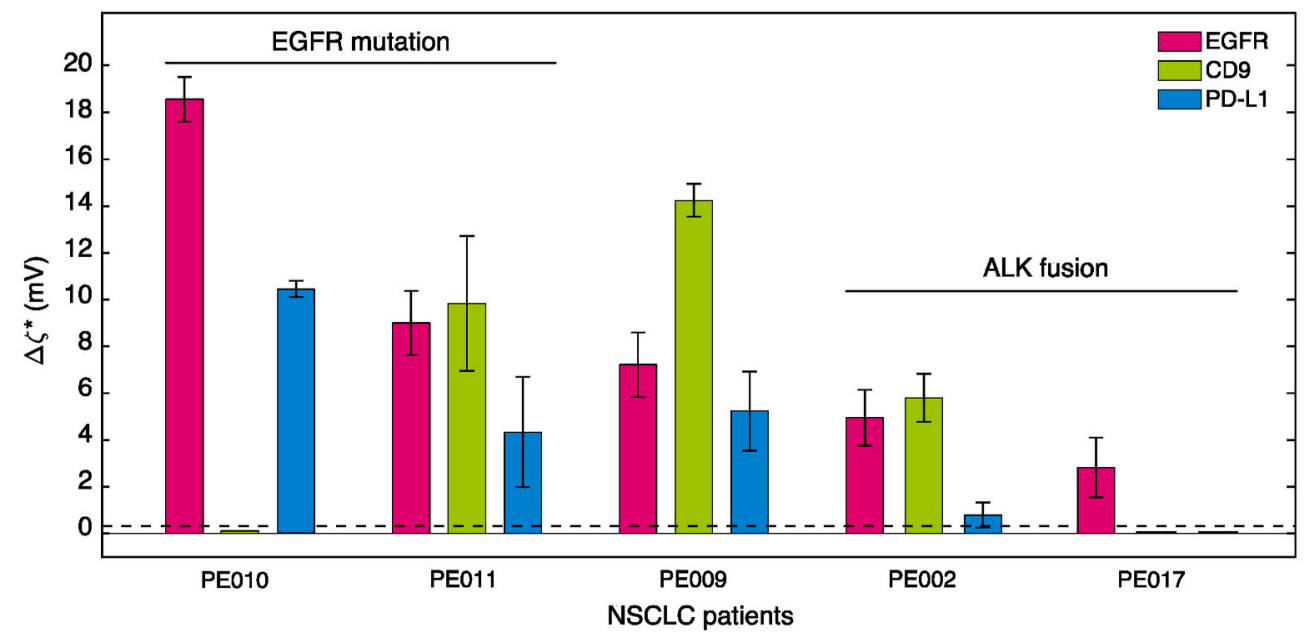

C

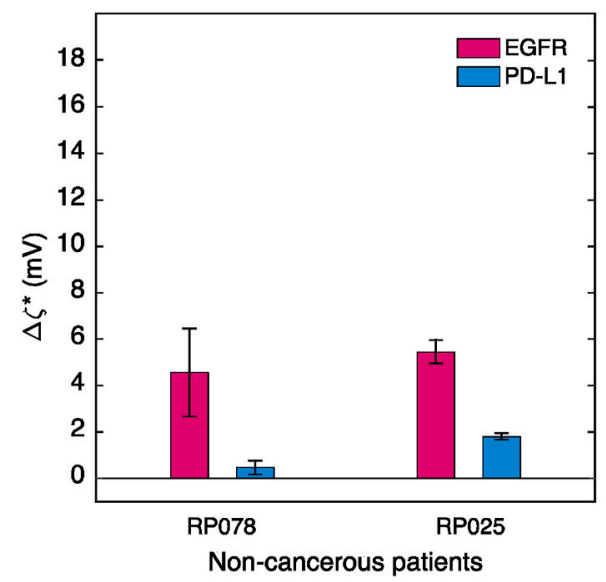

D

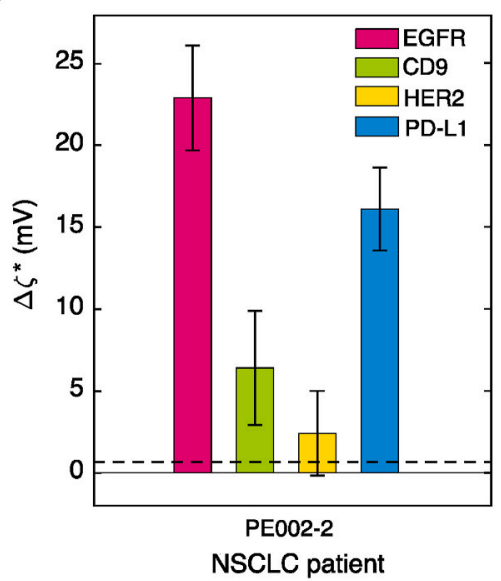

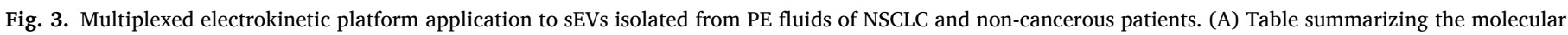

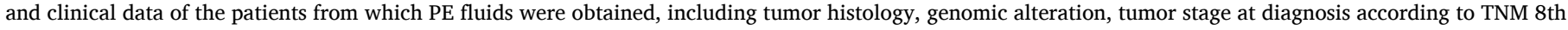

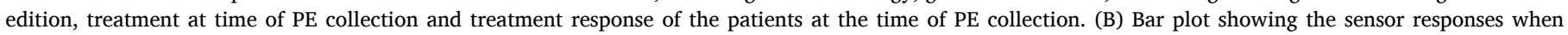

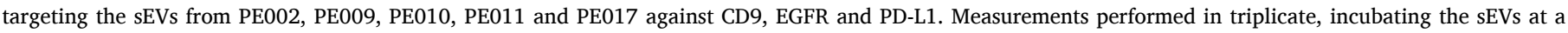

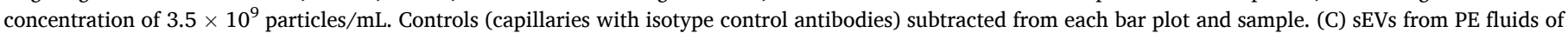

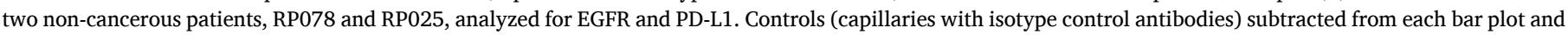

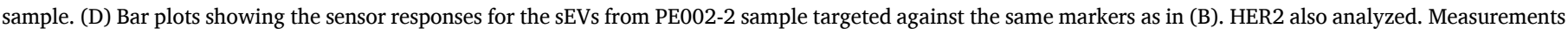
performed in duplicate. The dashed lines depict the MDS of the platform in its three-channel configuration measurement ( $\sim 0.4 \mathrm{mV})$.

majority of the patients, but with a differential expression among the samples. In particular, EGFR was found to be more abundant in PE010 and PE011 ( $\sim 18.5 \mathrm{mV}$ and $\sim 9 \mathrm{mV})$ as compared to PE002 and PE017 $(\sim 5 \mathrm{mV}$ and $\sim 2.8 \mathrm{mV})$. CD9 instead, seemed to be more abundant in PE009 $(\sim 14.2 \mathrm{mV})$ as compared to the other samples. The same trends were confirmed by WB profiling, which showed a higher expression level of both these markers on sEVs from PE011 and PE009 as compared to PE002 (Fig. S2 and (Stiller et al., 2021)). With respect to PD-L1, a higher electrokinetic response was evident in PE010 $(\sim 10.4 \mathrm{mV})$ as compared to PE011 ( $\sim 4.3 \mathrm{mV})$ and PE009 $(\sim 5.2 \mathrm{mV})$, while the signal was just above the MDS for the sEVs from PE002 ( $\sim 0.8 \mathrm{mV}$, Fig. 3B). The expression of PD-L1 was further analyzed on the same sEV batches using PEA analytics (Fig. S2), which also revealed similar differences in PD-L1 expression levels among the analyzed samples. The sEVs from PE fluids 
of the two non-cancerous patients were analyzed for EGFR and PD-L1, in order to investigate whether these markers showed significant differences as compared to the levels detected on sEVs from NSCLC patients. The results, reported in Fig. 3C, suggested very low levels of both markers, which were comparable to those detected in NSCLC patients with ALK fusions.

\subsection{Effect of treatment on sEV surface markers}

Finally, to examine the potentials of the electrokinetic method for treatment monitoring, we further analyzed the sEVs from the PE002 patient collected after 1-year on ALK-TKI crizotinib treatment, when the patient was clinically refractory. We refer to this sample as PE002-2 (Fig. 3A). Apart from compensatory mutations in the ALK kinase domain (Gristina et al., 2020), it is reported that ALK-TKI resistance also includes bypass signalling alterations in EGFR, IGF-1R and HER2 (Choi et al., 2017; Dagogo-Jack et al., 2017; Gristina et al., 2020; Minari et al., 2020). For this reason, we also included HER2 in addition to the previously studied markers CD9, EGFR and PD-L1 for profiling of sEVs from PE002-2. When HER2 was analyzed on the sEVs from the initial PE002 sample, the sensor did not show any response (data not shown). Fig. 3C presents the sensor responses for the markers analyzed on sEVs from PE002-2 patient. With respect to CD9, similar average values $(\sim 6 \mathrm{mV})$ were observed in sEVs from PE002-2 as compared to those detected at initial sample collection (PE002). On the contrary, EGFR and PD-L1 levels on sEVs significantly increased after crizotinib, showing a $\sim 4$.6-fold and a $\sim 21$-fold increase, respectively in PE002-2, as compared to their levels in sEVs from PE002. Finally, HER2 could be detected in PE002-2, despite showing a weak response $(\sim 2.5 \mathrm{mV})$.

\section{Discussion}

A key benefit of liquid biopsies is the ability to follow cancer patients during their treatments, thereby guiding the clinicians with effective therapy choices, such as precision cancer medicine agents or immune checkpoint inhibitors (ICI). This is important also in NSCLC treatment monitoring, where EGFR-TKI, ALK-TKI or PD-1/PD-L1 immune therapy have changed the treatment landscape, however responses of patients are often heterogenous (Abdel Karim and Kelly, 2019; Gristina et al., 2020; Lazzari et al., 2020). In clinical settings, circulating/cell-free DNA is partially used as a biomarker (BM) for evaluating treatment response when tumor biopsy is not feasible (Rolfo et al., 2018). However, DNA does not give information on alterations in tumor signalling taking place on mRNA or protein levels. In these cases, EVs from tumor cells per se as well as from immune cells within the tumor may offer a superior source of BMs. For analysis of such vesicles, the electrokinetic principle using streaming current represents a very promising method due to its simple design, good sensitivity and label-free approach. However, before us the principle was only applied for detection of proteins or small molecules. In the current study, we developed and validated a multiplexed electrokinetic platform for monitoring of multiple surface markers on sEVs and its application on clinical samples, e.g., EVs isolated from PE fluids of NSCLC patients with different genomic alterations/applied treatments and from non-cancerous patients.

In order to highlight the novelty of the study, we compared our work with reported articles both in the context of streaming current-based detection of bio-analytes and EV analysis. The comparisons are presented in Tables 1 and 2, respectively. Table 1 summarizes the reports on streaming current-based detection of biological targets. While the technique has been used for analysis of various bio-analytes, e.g. proteins, DNA etc., this is the first ever report demonstrating multiplexed detection and analysis of clinical sample. The comparison of LOD among the reported values also indicates an improvement over the previous reports. In addition, we have further compared our results on multiplexed EV profiling with other established methods such as SPR, electrochemical sensors as well as with some of the main fluidic-based

\section{Table 1}

Comparison of different works that have used the streaming current-based method for detection of biomolecules. References: (Cavallaro et al., 2019; Dev et al., 2016; Koch et al., 1999; Li et al., 2018; Martins et al., 2011; Sahu et al., 2021).

\begin{tabular}{|c|c|c|c|c|}
\hline Work & Target & LOD & Multiplexing & $\begin{array}{l}\text { Clinical } \\
\text { samples }\end{array}$ \\
\hline S. Koch et al. (1999) & Proteins & NA & No & No \\
\hline $\begin{array}{l}\text { D.C. Martins et al. } \\
\text { (2011) }\end{array}$ & DNA & NA & No & No \\
\hline Y. Li et al. (2018) & DNA & $10 \mathrm{nM}$ & No & No \\
\hline A. Dev et al. (2016) & Proteins & $100 \mathrm{pM}$ & No & No \\
\hline Sahu et al. (2021) & Proteins & $90 \mathrm{pM}$ & No & No \\
\hline $\begin{array}{l}\text { S. Cavallaro et al. } \\
\text { (2019) }\end{array}$ & EVs & $0.4 \mathrm{pM}$ & No & No \\
\hline Current work & EVs & $\begin{array}{l}<0.4 \\
\text { pM }\end{array}$ & Yes & Yes \\
\hline
\end{tabular}

detection methods (see Table 2). As seen in the table, for many matured technologies such as electrochemical sensing, SPR based detection etc., the capacity of multiplexed EV analysis and their application in clinical settings has only been demonstrated in recent past, despite the fact that such technologies have been extensively studied for many decades. Several fluidic-based techniques, e.g. based on lateral flow/inertial manipulation, are still limited to the analysis of cell line-derived samples. In this context, the successful demonstration of label-free multiplexed EV analysis from clinical sample is an important achievement for the relatively new method presented here. The current report also demonstrates the prospect of monitoring targeted cancer treatment by using EVs as a source of biomarkers. This aspect has been rarely addressed by the emerging methodologies including those relying on fluidic-based techniques.

\subsection{Multiplexed capability of the electrokinetic method}

As discussed earlier, the multiplexing capacity is one of the most important aspects of biosensor development and this aspect has been highlighted in a large number of research articles (Cho et al., 2019; Na et al., 2021; Shen et al., 2021; Zhang et al., 2021). It is well understood that practical application of biosensors would be seriously limited without this capacity. This is particularly true for cancer diagnosis using EVs, since several surface markers need to be analyzed from a small volume of samples (Cho et al., 2019; Fan et al., 2020). In the current design, this is achieved by simultaneous measurements on four microchannels that are connected in parallel, where each of them is functionalized to profile different surface markers. These channels share the same inlet flow (either buffer or sample) and a Pt electrode, while splitting afterwards, in order to allow independent current measurements. Therefore, the same original batch of sample loaded in the system is then equally distributed among the different channels. Conceptually, the multiplexing can also be achieved in a single channel, using a series configuration, as presented in Fig. S3. However, this strategy would require the immobilization of different antibodies on different parts of the channel and a separate set of electrodes for each of the parts. Such arrangements are difficult to achieve in a closed micro-capillary. Besides, there are other practical problems. For example, as the number of sensors increases, the length of the channel will proportionally increase, resulting in a very large pressure across the channel. Finally, as EVs co-express multiple markers on their surfaces, flowing the sample in a series configuration would cause its depletion over the different channels, due to capture of the vesicles by the sensors close to the inlet. For these reasons, we selected and optimized our multiplexed platform using a parallel configuration of the microcapillary sensors.

The data on the platform characterization and with sEVs isolated from cell culture media of NSCLC cells (Fig. 2) demonstrate that our electrokinetic sensor based on streaming current is suitable for multiplexed analysis of EV surface proteins. As shown in Fig. 2A and B, the 
Table 2

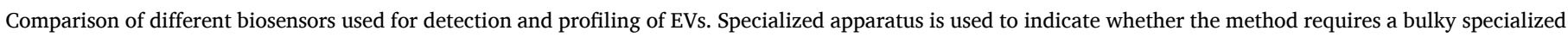

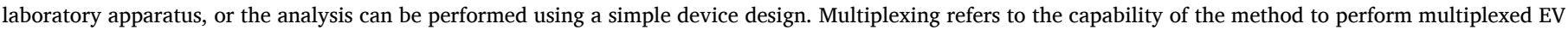

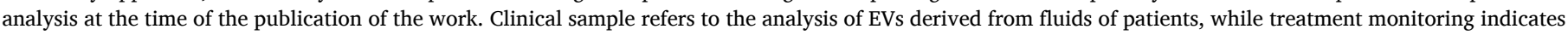

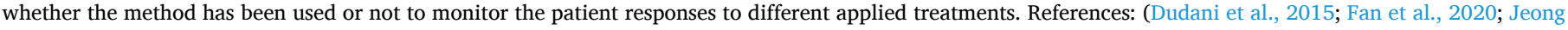
et al., 2016; Kanwar et al., 2014; Sina et al., 2016; Vaidyanathan et al., 2014; Yu et al., 2020).

\begin{tabular}{|c|c|c|c|c|c|c|c|}
\hline Work & Sensing method & Disease analyzed & Label-free & $\begin{array}{l}\text { Specialized } \\
\text { apparatus }\end{array}$ & Multiplexing & $\begin{array}{l}\text { Clinical } \\
\text { samples }\end{array}$ & $\begin{array}{l}\text { Treatment } \\
\text { monitoring }\end{array}$ \\
\hline S. Jeong et al. (2016) & Electrochemical & Ovarian cancer & No, enzyme & No & Yes & Yes & Yes \\
\hline A. Sina et al. (2016) & SPR & Breast cancer & Yes & No & Yes & Yes & No \\
\hline $\begin{array}{l}\text { R. Vaidya-nathan et al. } \\
\text { (2014) }\end{array}$ & Nanoshearing & $\begin{array}{l}\text { Breast/Prostate } \\
\text { cancer }\end{array}$ & No, fluorophores & No & Yes & Yes & No \\
\hline S. Kanwar al. (2014) & Optical & Pancreatic cancer & No, fluorophores & No & No & Yes & No \\
\hline Q. Yu et al. (2020) & Lateral flow/Absorbance & NSCLC & No, Au particles & No & No & No & No \\
\hline Y. Fan et al. (2020) & SPR & NSCLC & Yes & No & Yes & Yes & No \\
\hline J. Dudani et al. (2015) & $\begin{array}{l}\text { Inertial manipulation/ } \\
\text { Optical }\end{array}$ & $\begin{array}{l}\text { Melanoma/breast } \\
\text { cancer }\end{array}$ & $\begin{array}{l}\text { No, fluorophores/ } \\
\text { beads }\end{array}$ & Yes & No & No & No \\
\hline Current work & $\begin{array}{l}\text { Electrokinetic/streaming } \\
\text { current }\end{array}$ & NSCLC & Yes & No & Yes & Yes & Yes \\
\hline
\end{tabular}

measurements parameters do not change significantly upon simultaneous connection of 4 microcapillary sensors. In particular, Fig. $2 \mathrm{~A}$ indicates that, despite a $\sim 2$-fold increase when connecting more than 2 channels, the current noise still remains low $(<0.3 \mathrm{pA})$ for three- and four-channel configurations, as compared to the detected signals ( $>25-30 \mathrm{pA}$, data not shown). Such increased noise levels might come from the electrical measurement and/or from the fluidic part, due to flow splitting and distribution at the multiport connector. Variations in the $\zeta^{*}$ baselines, as those reported in Fig. 2B, might also be caused by small changes in the flow of one channel upon connection of the others. However, all such $\zeta^{*}$ changes (Fig. 2B) are in the order of the MDSs of the corresponding number of channels (Fig. 2A) and therefore can be neglected. Overall, these data suggest that the measurement outcomes do not change whether a marker is measured alone or in combination with others in parallel. Here, we would also like to emphasize that we tested a maximum of 4 channels only to validate the proof of concept for multiplexed detection using the streaming current method. The presented setup utilizes a commercial manifold which can accommodate up to 4 parallel microcapillaries without significant variations in the flowrates (see Fig. S4) in the channels. In principle, the method can be extended to perform parallel measurements on a much higher number of channels for simultaneous detection of a large cohort of analytes. Such a capacity will be extremely valuable, particularly for cancer diagnosis where multiple pathways might co-exist in tumor progression, requiring the detection of several markers (Guo et al., 2020). Fig. 2C demonstrates the successful application of the multiplexed electrokinetic platform for analysis of sEV surface markers. The results also suggest that the signals can be enhanced by optimizing the capture probes, as also reported in our previous study (Sahu et al., 2020) and in Supplementary Information (Fig. S7). The aspect of signal enhancement is very important in biosensing, as also discussed by others (Peteu, 2010), as it can lead to potentially lower LOD and enhanced sensitivity. The low signals obtained for HER2 can be explained by a very low surface expression level of the marker in these sEVs (as compared to other sEVs and/or tumor receptors) and/or by a low affinity of the capture probe used. On the other hand, the measurements with sEVs from NSCLC cells suggested the presence of slight differences in the flow profiles ( $\Delta$ flowrates for the applied pressures of 1.5 and 3 bar) among the different channels when connecting 4 capillaries simultaneously. This effect might be caused by differences in the fluidic resistances and/or flaws in the multiport connector that distributes the flow among the channels. Therefore, for the present investigation, we decided to measure the sEVs from the malignant PE fluids of patients with the platform in its three-channel configuration. Furthermore, the measurements were performed with sEVs at a concentration of $3.5 \times 10^{9}$ particles $/ \mathrm{mL}$ and required $\sim 20 \mu \mathrm{L}$ of sample per microcapillary sensor. This corresponded to a total sample volume of $\sim 110 \mu \mathrm{L}$ for a four-channel configuration platform (sensors + dead volume in microfluidic connections), and therefore a total of $3.8 \times$ $10^{8} \mathrm{sEVs}$. For the PE samples used in the present study, EVs were isolated from 0.3 to $5 \mathrm{ml}$ of filtered PE fluid. The number of EVs per $\mathrm{ml}$ of PE fluid was $\sim 1 \times 10^{10}$, meaning that only $<30-40 \mu \mathrm{L}$ of liquid is necessary to perform such a multiplexed measurement with the proposed electrokinetic platform. Moreover, the figure of merit of our sensor, defined as the LOD, has been demonstrated to be $2 \times 10^{8}$ particles $/ \mathrm{mL}$, corresponding to $0.4 \mathrm{pM}$, (Cavallaro et al., 2019) when targeting EGFR. Considering the higher EGFR signal detected for the same concentration in the current study (Fig. 2C), our sensor is expected to have a LOD $<2 \times$ $10^{8}$ particles/mL, thus covering the entire range of EVs that can be present in body fluids $\left(1 \times 10^{8}-3 \times 10^{12} \mathrm{EV} / \mathrm{mL}\right)$ (Ricklefs et al., 2019; Sina et al., 2016). Overall, the data in Fig. 2 demonstrate the capability of our electrokinetic platform to measure multiple EV markers simultaneously from a low sample volume with a high specificity and reproducibility.

\subsection{Analysis of sEVs from clinical samples}

The results on the clinical samples (Fig. 3) demonstrate the possibility to use the platform for detection and treatment monitoring of sEVs collected from malignant PE fluids of advanced NSCLC patients. A challenge arising when analyzing patient samples from liquid biopsies can be the presence of proteins and/or analytes that might get coisolated with EVs and interact with the biosensors, thereby causing higher NSB as compared to cell line derived samples. However, our data suggest that, despite a 4-20-fold increase as compared to cell line derived vesicles, the sensor responses for control measurements on sEVs from patient liquid biopsies were still significantly lower than those detected for the different EV markers. A possible explanation might be derived from the fundamental principle of the sensing method, which strongly depends on the size of the analyte, as shown in our previous report (Sahu et al., 2020). Therefore, an EV, being much larger than a free protein, is expected to produce a stronger electrokinetic signal. Since the PE samples were collected from patients with different genomic drivers of their tumors, e.g., EGFR, ALK and KRAS, whom also have been given different treatment regimens including EGFR- and ALK-TKIs (Fig. 3A), we expected different expression levels of the analyzed markers and therefore different responses of the multiplexed electrokinetic sensors. The results confirmed the expectations. For example, the sEVs from PE010 and PE011, which were obtained from patients with EGFR-mutant NSCLC, showed a stronger EGFR signal as compared to the sEVs from PE009, PE002 and PE017, which were instead collected from patients with a KRAS mutation and an ALK-driven tumor, respectively. With respect to CD9, PE010 and PE017 showed the lowest levels among the analyzed PE fluids. The analyses of EGFR and CD9 on PE002, PE009 and PE011 by WB ((Stiller et al., 2021) and 
Fig. S2) confirmed the same trends, highlighting that our electrokinetic data is in line with the results obtained by standard techniques.

In our study, we also analyzed PD-L1, an important ICI target in NSCLC, which has also been found in SEVs from NSCLC patient liquid biopsies (D. H. Kim et al., 2019). While the electrokinetic sensors detected a clear signal from PD-L1 on sEVs from PE010, PE011 and PE009, the vesicles from PE002 and PE017 showed instead very weak levels. When PD-L1 expression was evaluated with PEA analytics, there was a clear difference in expression among PE002, PE009 and PE011 sEVs, confirming the sensor responses (Fig. S2). These results suggest different PD-L1 levels on sEVs from malignant PE fluid samples that can also be captured by our electrokinetic sensors. Moreover, the results on the PE samples from non-cancerous patients suggested decreased expression levels of the analyzed markers as compared to most of the NSCLC patients (PE010, PE011 and PE009), while showing similar levels as those detected in patients with ALK fusion tumors.

\subsection{Use of the multiplexed platform for treatment monitoring}

Finally, the results obtained from PE002 patient prior and post ALKTKI crizotinib hold promise for the application of the electrokinetic method for tumor treatment monitoring based on sEVs from liquid biopsies. In particular, the sensors showed higher EGFR and PD-L1 signals at the time point of ALK-TKI crizotinib refractoriness (PE002-2) than early upon treatment induction (PE002), suggesting that crizotinib treatment might cause increased expressions of both these receptors in sEVs. HER2 also showed a higher response than that detected in PE002, yet the increase was less prominent ( $\sim 2$-fold). Such increased EGFR or HER2 expression/functional dependency has been reported in literature for ALK-TKI refractory NSCLC cells and patients (Dagogo-Jack et al., 2017; Gristina et al., 2020; Minari et al., 2020). Albeit ICI treatment of EGFR- or ALK-driven NSCLC has been reported to have lower efficacy than in NSCLC in general (Gainor et al., 2016), it has been shown that PD-L1 expression is increased in ALK-TKI refractory NSCLC cells in vitro (S. J. Kim et al., 2019). Interestingly, the electrokinetic sensors also detected significantly increased levels of PD-L1 on the sEVs from the ALK-TKI refractory patient PE002-2. High levels of PD-L1 were also detected on the sEVs from the EGFR-TKI refractory patient PE010 (Fig. 3B), reinforcing the possibility of an overexpression of this marker in case of treatment refractoriness. However, further analyses of sEVs from a larger cohort of patients, in general, and ALK-TKI treated patients, in particular, are needed to make general conclusions on possible cancer resistance mechanisms based on EV measurements.

\section{Conclusions}

In conclusion, in this study we first demonstrated the possibility to use the electrokinetic method to perform multiplexed detection and profiling of sEV surface proteins. The results on the platform characterization indicated that the device performances in the multiplexed configuration remain similar in terms of noise, detection sensitivity and reproducibility. The results on the vesicles isolated from both the malignant PE fluids of the NSCLC patients with different genomic alterations (ALK, KRAS or EGFR) and from the fluids of non-cancerous individuals demonstrated the capability of the electrokinetic sensors to profile several tumor cell surface markers on EVs from clinical samples. The surface marker profiles of the EVs extracted from the patients show expected trends, as verified by other complementary methods, such as WB and PEA. Although a larger patient cohort is necessary to state any clear conclusion on the relation between the analyzed proteins and the possible cancer resistance mechanisms, the data indicated interpatient differences in accordance with other investigations. With the demonstration of multiplexed detection and analysis of clinical samples, the platform presented here shows important improvements over the previous reports employing the same detection principle, and thus can be translated for clinical application.

\section{Author contributions}

SC: conceptualization, data curation, formal analysis, investigation, validation, visualization, writing - original draft, writing - review and editing, PH: data curation, formal analysis, resources, writing - review and editing, SSS: data curation, formal analysis, software, writing - review and editing, LB: data curation, VK: data curation, writing - review and editing, SE: resources, investigation, project administration, writing - review and editing, RL: resources, funding acquisition, project administration, JL: funding acquisition, project administration, writing - review and editing, KV: formal analyses, resources, funding acquisition, writing - original draft, writing - review and editing, AD: method development and conceptualization, supervision and funding acquisition, project administration and writing - original draft, writing - review and editing.

\section{CRediT authorship contribution statement}

Sara Cavallaro: Conceptualization, Data curation, Formal analysis, Investigation, Validation, Visualization, Writing - original draft, Writing - review \& editing. Petra Hååg: Data curation, Formal analysis, Resources, Writing - review \& editing. Siddharth S. Sahu: Data curation, Formal analysis, Software, Writing - review \& editing. Lorenca Berisha: Data curation. Vitaliy O. Kaminskyy: Data curation, Writing review \& editing. Simon Ekman: Resources, Investigation, Project administration, Writing - review \& editing. Rolf Lewensohn: Resources, Funding acquisition, Project administration. Jan Linnros: Funding acquisition, Project administration, Writing - review \& editing. Kristina Viktorsson: Formal analysis, Resources, Funding acquisition, Writing - original draft, Writing - review \& editing. Apurba Dev: Methodology, Conceptualization, Supervision, Funding acquisition, Project administration, Writing - original draft, Writing - review \& editing.

\section{Declaration of competing interest}

The authors declare that they have no known competing financial interests or personal relationships that could have appeared to influence the work reported in this paper.

\section{Acknowledgements}

This study was supported by grants from the Erling Persson Family Foundation, Stockholm Cancer Society (\#171123, \#191293, \#201202) the Swedish Cancer Society (CAN 2015/401; CAN 2018/597), Stockholm County Council (\#20160287, \#20180404), The Research Council (\#2016-05051) and funds of Karolinska University Hospital FOUU (\#75032.). For the tumor related part, the support and help from MSc Vasiliki Arapi, Dr Caroline Kamali, Dr Luigi De Petris, Dr Metka Novak and Dr Per Hydbring is acknowledged. The Clinical biomarker facility at SciLife Lab Sweden is acknowledged for providing assistance in PEA protein analyses.

\section{Appendix A. Supplementary data}

Supplementary data to this article can be found online at https://doi. org/10.1016/j.bios.2021.113568.

\section{References}

Abdel Karim, N., Kelly, K., 2019. Role of targeted therapy and immune checkpoint blockers in advanced non-small cell lung cancer: a review. Oncol. 24, 1270-1284. https://doi.org/10.1634/theoncologist.2018-0112.

Adamczyk, Z., Sadlej, K., Wajnryb, E., Nattich, M., Ekiel-Jezewska, M.L., Bławzdziewicz, J., 2010. Streaming potential studies of colloid, polyelectrolyte and protein deposition. Adv. Colloid Interface Sci. 153, 1-29. https://doi.org/10.1016/j. cis.2009.09.004. 
An, T., Qin, S., Sun, D., Huang, Y., Hu, Y., Li, S., Zhang, H., Li, B., Situ, B., Lie, L., Wu, Y., Zheng, L., 2019. Unique protein profiles of extracellular vesicles as diagnostic biomarkers for early and advanced non-small cell lung cancer. Proteomics 19. https://doi.org/10.1002/pmic.201800160.

Baburaj, G., Damerla, R.R., Udupa, K.S., Parida, P., Munisamy, M., Kolesar, J., Rao, M., 2020. Liquid biopsy approaches for pleural effusion in lung cancer patients. Mol. Biol. Rep. 47, 8179-8187. https://doi.org/10.1007/s11033-020-05869-7.

Cavallaro, S., Hååg, P., Viktorsson, K., Krozer, A., Fogel, K., Lewensohn, R., Linnros, J., Dev, A., 2021. Comparison and optimization of nanoscale extracellular vesicle imaging by scanning electron microscopy for accurate size-based profiling and morphological analysis. Nanoscale Adv. 3, 3053-3063. https://doi.org/10.1039/ d0na00948b.

Cavallaro, S., Horak, J., Hååg, P., Gupta, D., Stiller, C., Sahu, S.S., Görgens, A., Gatty, H. K., Viktorsson, K., El Andaloussi, S., Lewensohn, R., Karlstrom, A.E., Linnros, J., Dev, A., 2019. Label-free surface protein profiling of extracellular vesicles by an electrokinetic sensor. ACS Sens. 4, 1399-1408. https://doi.org/10.1021/ acssensors.9b00418.

Cho, S., Yang, H.C., Rhee, W.J., 2019. Simultaneous multiplexed detection of exosomal microRNAs and surface proteins for prostate cancer diagnosis. Biosens. Bioelectron. 146, 111749. https://doi.org/10.1016/j.bios.2019.111749.

Choi, S.H., Kim, D.H., Choi, Y.J., Kim, S.Y., Lee, J.E., Sung, K.J., Kim, W.S., Choi, C.M., Rho, J.K., Lee, J.C., 2017. Multiple receptor tyrosine kinase activation related to ALK inhibitor resistance in lung cancer cells with ALK rearrangement. Oncotarget 8, 58771-58780. https://doi.org/10.18632/oncotarget.17680.

Dagogo-Jack, I., Fabrizio, D., Lennerz, J., Schrock, A.B., Young, L., Mino-Kenudson, M., Digumarthy, S.R., Heist, R.S., Ali, S.M., Miller, V.A., Shaw, A.T., 2017. Circulating tumor DNA identifies EGFR coamplification as a mechanism of resistance to crizotinib in a patient with advanced MET-amplified lung adenocarcinoma. J. Thorac. Oncol. 12, e155-e157. https://doi.org/10.1016/j.jtho.2017.04.023.

De Mattos-Arruda, L., Siravegna, G., 2021. How to use liquid biopsies to treat patients with cancer. ESMO Open 6, 100060. https://doi.org/10.1016/j. esmoop.2021.100060.

Dev, A., Horak, J., Kaiser, A., Yuan, X., Perols, A., Björk, P., Karlström, A.E., Kleimann, P., Linnros, J., 2016. Electrokinetic effect for molecular recognition: a label-free approach for real-time biosensing. Biosens. Bioelectron. 82, 55-63. https://doi.org/10.1016/j.bios.2016.03.060.

Dudani, J.S., Gossett, D.R., Tse, H.T.K., Lamm, R.J., Kulkarni, R.P., Di, D., 2015. Rapid inertial solution exchange for enrichment and flow cytometric detection of microvesicles. Biomicrofluidics 9 (1-9), 014112. https://doi.org/10.1063/ 1.4907807.

Fan, Y., Duan, X., Zhao, M., Wei, X., Wu, J., Chen, W., Liu, P., Cheng, W., Cheng, Q., Ding, S., 2020. High-sensitive and multiplex biosensing assay of NSCLC-derived exosomes via different recognition sites based on SPRi array. Biosens. Bioelectron. 154, 112066. https://doi.org/10.1016/j.bios.2020.112066.

Ferguson, S., Weissleder, R., 2020. Modeling EV kinetics for use in early cancer detection. Adv. Biosyst. 4, 1-11. https://doi.org/10.1002/adbi.201900305.

Gainor, J.F., Shaw, A.T., Sequist, L.V., Fu, X., Azzoli, C.G., Piotrowska, Z., Huynh, T.G., Zhao, L., Fulton, L., Schultz, K.R., Howe, E., Farago, A.F., Sullivan, R.J., Stone, J.R., Digumarthy, S., Moran, T., Hata, A.N., Yagi, Y., Yeap, B.Y., Engelman, J.A., MinoKenudson, M., 2016. EGFR mutations and ALK rearrangements are associated with low response rates to PD-1 pathway blockade in non-small cell lung cancer: a retrospective analysis. Clin. Canc. Res. 22, 4585-4593. https://doi.org/10.1158/ 1078-0432.CCR-15-3101.

Gristina, V., Mantia, M. La, Iacono, F., Galvano, A., Russo, A., Bazan, V., 2020. The emerging therapeutic landscape of alk inhibitors in non-small cell lung cancer. Pharmaceuticals 13, 1-23. https://doi.org/10.3390/ph13120474.

Guo, Y., Song, J., Wang, Y., Huang, L., Sun, L., Zhao, J., Zhang, S., Jing, W., Ma, J., Han, C., 2020. Concurrent genetic alterations and other biomarkers predict treatment efficacy of EGFR-TKIs in EGFR-mutant non-small cell lung cancer: a review. Front. Oncol. 10 https://doi.org/10.3389/fonc.2020.610923.

Hoshino, A., Kim, H.S., Bojmar, L., Gyan, K.E., Cioffi, M., Hernandez, J., Zambirinis, C.P., Rodrigues, G., Molina, H., Heissel, S., Mark, M.T., Steiner, L., Benito-Martin, A., Lucotti, S., Di Giannatale, A., Offer, K., Nakajima, M., Williams, C., Nogués, L., Pelissier Vatter, F.A., Hashimoto, A., Davies, A.E., Freitas, D., Kenific, C.M., Ararso, Y., Buehring, W., Lauritzen, P., Ogitani, Y., Sugiura, K., Takahashi, N., Alečković, M., Bailey, K.A., Jolissant, J.S., Wang, H., Harris, A., Schaeffer, L.M., García-Santos, G., Posner, Z., Balachandran, V.P., Khakoo, Y., Raju, G.P., Scherz, A., Sagi, I., Scherz-Shouval, R., Yarden, Y., Oren, M., Malladi, M., Petriccione, M., De Braganca, K.C., Donzelli, M., Fischer, C., Vitolano, S., Wright, G.P., Ganshaw, L., Marrano, M., Ahmed, A., DeStefano, J., Danzer, E., Roehrl, M.H.A., Lacayo, N.J., Vincent, T.C., Weiser, M.R., Brady, M.S., Meyers, P.A., Wexler, L.H., Ambati, S.R., Chou, A.J., Slotkin, E.K., Modak, S., Roberts, S.S., Basu, E.M., Diolaiti, D., Krantz, B. A., Cardoso, F., Simpson, A.L., Berger, M., Rudin, C.M., Simeone, D.M., Jain, M., Ghajar, C.M., Batra, S.K., Stanger, B.Z., Bui, J., Brown, K.A., Rajasekhar, V.K., Healey, J.H., de Sousa, M., Kramer, K., Sheth, S., Baisch, J., Pascual, V., Heaton, T.E., La Quaglia, M.P., Pisapia, D.J., Schwartz, R., Zhang, H., Liu, Y., Shukla, A., Blavier, L., DeClerck, Y.A., LaBarge, M., Bissell, M.J., Caffrey, T.C., Grandgenett, P. M., Hollingsworth, M.A., Bromberg, J., Costa-Silva, B., Peinado, H., Kang, Y., Garcia, B.A., O'Reilly, E.M., Kelsen, D., Trippett, T.M., Jones, D.R., Matei, I.R., Jarnagin, W.R., Lyden, D., 2020. Extracellular vesicle and particle biomarkers define multiple human cancers. Cell 182, 1044-1061. https://doi.org/10.1016/j. cell.2020.07.009 e18.

Hydbring, P., De Petris, L., Zhang, Y., Brandén, E., Koyi, H., Novak, M., Kanter, L., Hååg, P., Hurley, J., Tadigotla, V., Zhu, B., Skog, J., Viktorsson, K., Ekman, S., Lewensohn, R., 2018. Exosomal RNA-profiling of pleural effusions identifies adenocarcinoma patients through elevated miR-200 and LCN2 expression. Lung Canc. 124, 45-52. https://doi.org/10.1016/j.lungcan.2018.07.018.

Jeong, S., Park, J., Pathania, D., Castro, C.M., Weissleder, R., Lee, H., 2016. Integrated magneto-electrochemical sensor for exosome analysis. ACS Nano 10, 1802-1809. https://doi.org/10.1021/acsnano.5b07584.

Kang, M., Park, C., Kim, S.H., Yoon, S.W., Suh, K.J., Kim, Y.J., Ock, C., Kim, M., Keam, B., Kim, T.M., Kim, D., Heo, D.S., Lee, J.S., 2021. Programmed Death-Ligand 1 Expression Level as a Predictor of EGFR Tyrosine Kinase Inhibitor Efficacy in Lung Adenocarcinoma, 1, pp. 699-711. https://doi.org/10.21037/tlcr-20-893.

Kanwar, S.S., Dunlay, J., Simeone, M., Nagrath, S., 2014. Microfluidic device (ExoChip) for on-chip isolation, quantification and characterization of circulating exosomes. Lab Chip 14, 1891-1900. https://doi.org/10.1039/c4lc00136b.

Kim, D.H., Kim, H.R., Choi, Y.J., Kim, S.Y., Lee, J.E., Sung, K.J., Sung, Y.H., Pack, C.G., Jung, M. kyo, Han, B., Kim, K., Kim, W.S., Nam, S.J., Choi, C.M., Yun, M., Lee, J.C., Rho, J.K., 2019. Exosomal PD-L1 promotes tumor growth through immune escape in non-small cell lung cancer. Exp. Mol. Med. 51 https://doi.org/10.1038/s12276-0190295-2.

Kim, S.J., Kim, S., Kim, D.W., Kim, M., Keam, B., Kim, T.M., Lee, Y., Koh, J., Jeon, Y.K., Heo, D.S., 2019a. Alterations in PD-L1 expression associated with acquisition of resistance to ALK inhibitors in ALK-rearranged lung cancer. Cancer Res. Treat 51, 1231-1240. https://doi.org/10.4143/crt.2018.486.

Kirby, B.J., Hasselbrink, E.F., 2004. Zeta potential of microfluidic substrates: 1. Theory, experimental techniques, and effects on separations. Electrophoresis 25, 187-202. https://doi.org/10.1002/elps.200305754.

Koch, S., Woias, P., Meixner, L.K., Drost, S., Wolf, H., 1999. Protein detection with a novel ISFET-based zeta potential analyzer. Biosens. Bioelectron. 14, 413-421.

König, D., Prince, S.S., Rothschild, S.I., 2021. Targeted therapy in advanced and metastatic non-small cell lung cancer. An update on treatment of the most important actionable oncogenic driver alterations. Cancers 13, 804. https://doi.org/10.3390/ cancers13040804.

Krug, A.K., Enderle, D., Karlovich, C., Priewasser, T., Bentink, S., Spiel, A., Brinkmann, K., Emenegger, J., Grimm, D.G., Castellanos-Rizaldos, E., Goldman, J. W., Sequist, L.V., Soria, J.C., Camidge, D.R., Gadgeel, S.M., Wakelee, H.A., Raponi, M., Noerholm, M., Skog, J., 2018. Improved EGFR mutation detection using combined exosomal RNA and circulating tumor DNA in NSCLC patient plasma. Ann. Oncol. 29, 700-706. https://doi.org/10.1093/annonc/mdx765.

Lai-Kwon, J., Tiu, C., Pal, A., Khurana, S., Minchom, A., 2021. Moving beyond epidermal growth factor receptor resistance in metastatic non-small cell lung cancer - a drug development perspective. Crit. Rev. Oncol. Hematol. 159, 103225. https://doi.org/ 10.1016/j.critrevonc.2021.103225.

Lazzari, C., Gregorc, V., Karachaliou, N., Rosell, R., Santarpia, M., 2020. Mechanisms of resistance to osimertinib. J. Thorac. Dis. 12, 2851-2858. https://doi.org/10.21037/ jtd.2019.08.30.

Li, M.Y., Liu, L.Z., Dong, M., 2021. Progress on pivotal role and application of exosome in lung cancer carcinogenesis, diagnosis, therapy and prognosis. Mol. Canc. 20, 1-22. https://doi.org/10.1186/s12943-021-01312-y.

Li, Y., Lai, S.N., Zheng, B., 2018. A microfluidic streaming potential analyzer for labelfree DNA detection. Sensor. Actuator. B Chem. 259, 871-877. https://doi.org/ 10.1016/j.snb.2017.12.130.

Liu, J., Itchins, M., Nagrial, A., Cooper, W.A., De Silva, M., Barnet, M., Varikatt, W., Sivasubramaniam, V., Davis, A., Gill, A.J., Blinman, P., Lee, K., Hui, R., Gao, B., Pavlakis, N., Clarke, S., Lee, J., Boyer, M., Kao, S., 2021. Relationship between PD-L1 expression and outcome in EGFR-mutant lung cancer patients treated with EGFR tyrosine kinase inhibitors. Lung Canc. 155, 28-33. https://doi.org/10.1016/j. lungcan.2021.03.004.

Martins, D.C., Chu, V., Prazeres, D.M.F., Conde, J.P., 2011. Electrical detection of DNA immobilization and hybridization by streaming current measurements in microchannels. Appl. Phys. Lett. (1-3), 183702 https://doi.org/10.1063/1.3658457.

Minari, R., Gnetti, L., Lagrasta, C.A., Squadrilli, A., Bordi, P., Azzoni, C., Bottarelli, L., Cosenza, A., Ferri, L., Caruso, G., Silini, E.M., Tiseo, M., 2020. Emergence of a HER2amplified clone during disease progression in an ALK-rearranged NSCLC patient treated with ALK-inhibitors: a case report. Transl. Lung Cancer Res. 9, 787-792. https://doi.org/10.21037/tlcr.2020.04.03.

Na, H.K., Shon, H.K., Son, H.Y., Jang, E., Joh, S., Huh, Y.M., Castner, D.G., Lee, T.G., 2021. Utilization of chromogenic enzyme substrates for signal amplification in multiplexed detection of biomolecules using surface mass spectrometry. Sensor. Actuator. B Chem. 332, 129452. https://doi.org/10.1016/j.snb.2021.129452.

Pao, W., Miller, V.A., Politi, K.A., Riely, G.J., Somwar, R., Zakowski, M.F., Kris, M.G., Varmus, H., 2005. Acquired resistance of lung adenocarcinomas to gefitinib or erlotinib is associated with a second mutation in the EGFR kinase domain. PLoS Med. 2, 225-235. https://doi.org/10.1371/journal.pmed.0020073.

Pasini, L., Ulivi, P., 2020. Extracellular vesicles in non-small-cell lung cancer: functional role and involvement in resistance to targeted treatment and immunotherapy. Cancers 12. https://doi.org/10.3390/cancers12010040.

Peteu, S.F., 2010. Micro- to nano- Biosensors and actuators integrated for responsive delivery of countermeasures. Proc. Int. Semicond. Conf. CAS 1, 179-190. https:// doi.org/10.1109/SMICND.2010.5650417.

Planchard, D., Popat, S., Kerr, K., Novello, S., Smit, E.F., Faivre-Finn, C., Mok, T.S., Reck, M., Van Schil, P.E., Hellmann, M.D., Peters, S., 2018. Metastatic non-small cell lung cancer: ESMO Clinical Practice Guidelines for diagnosis, treatment and followup. Ann. Oncol. 29, iv192-iv237. https://doi.org/10.1093/annonc/mdy275.

Ricklefs, F.L., Maire, C.L., Reimer, R., Dührsen, L., et al., 2019. Imaging flow cytometry facilitates multiparametric characterization of extracellular vesicles in malignant brain tumours.pdf. J. Extracell. Vesicles 8 (2-18), 1588555.

Rolfo, C., Mack, P.C., Scagliotti, G.V., Baas, P., Barlesi, F., Bivona, T.G., Herbst, R.S., Mok, T.S., Peled, N., Pirker, R., Raez, L.E., Reck, M., Riess, J.W., Sequist, L.V., 
Shepherd, F.A., Sholl, L.M., Tan, D.S.W., Wakelee, H.A., Wistuba, I.I., Wynes, M.W., Carbone, D.P., Hirsch, F.R., Gandara, D.R., 2018. Liquid biopsy for advanced nonsmall cell lung cancer (NSCLC): a statement paper from the iaslc. J. Thorac. Oncol. 13, 1248-1268. https://doi.org/10.1016/j.jtho.2018.05.030.

Sahu, S.S., Stiller, C., Cavallaro, S., Karlström, A.E., Linnros, J., Dev, A., 2020. Influence of molecular size and zeta potential in electrokinetic biosensing. Biosens. Bioelectron. 152 https://doi.org/10.1016/j.bios.2020.112005.

Sahu, S.S., Stiller, C., Gomero, E.P., Nagy, Á., Karlström, A.E., Linnros, J., Dev, A., 2021. Electrokinetic sandwich assay and DNA mediated charge amplification for enhanced sensitivity and specificity. Biosens. Bioelectron. 176 https://doi.org/10.1016/j. bios.2020.112917.

Sandfeld-Paulsen, B., Aggerholm-Pedersen, N., Bæk, R., Jakobsen, K.R., Meldgaard, P., Folkersen, B.H., Rasmussen, T.R., Varming, K., Jørgensen, M.M., Sorensen, B.S., 2016. Exosomal proteins as prognostic biomarkers in non-small cell lung cancer. Mol. Oncol. 10, 1595. https://doi.org/10.1016/j.molonc.2016.10.003.

Santarpia, M., Liguori, A., D’Aveni, A., Karachaliou, N., Gonzalez-Cao, M., Daffinà, M.G. Lazzari, C., Altavilla, G., Rosell, R., 2018. Liquid biopsy for lung cancer early detection. J. Thorac. Dis. 10, S882-S897. https://doi.org/10.21037/jtd.2018.03.81.

Shen, Y., Modha, S., Tsutsui, H., Mulchandani, A., 2021. An origami electrical biosensor for multiplexed analyte detection in body fluids. Biosens. Bioelectron. 171, 112721. https://doi.org/10.1016/j.bios.2020.112721.

Sina, A.A.I., Vaidyanathan, R., Dey, S., Carrascosa, L.G., Shiddiky, M.J.A., Trau, M., 2016. Real time and label free profiling of clinically relevant exosomes. Sci. Rep. 6 , 1-9. https://doi.org/10.1038/srep30460.

Stiller, C., Viktorsson, K., Gomero, E.P., Hååg, P., Arapi, V., Kaminskyy, V.O., Kamali, C., De Petris, L., Ekman, S., Lewensohn, R., Karlström, A.E., 2021. Detection of tumorassociated membrane receptors on extracellular vesicles from non-small cell lung cancer patients via immuno-pcr. Cancers 13, 1-21. https://doi.org/10.3390/ cancers13040922.

Suda, K., Rivard, C.J., Mitsudomi, T., Hirsch, F.R., 2017. Overcoming resistance to EGFR tyrosine kinase inhibitors in lung cancer, focusing on non-T790M mechanisms. Expert Rev. Anticancer Ther. 17, 779-786. https://doi.org/10.1080/ 14737140.2017.1355243.

Sung, H., Ferlay, J., Siegel, R.L., Laversanne, M., Soerjomataram, I., Jemal, A., Bray, F., 2021. Global cancer statistics 2020: GLOBOCAN estimates of incidence and mortality worldwide for 36 cancers in 185 countries. CA A Cancer J. Clin. 1-41. https://doi.org/10.3322/caac.21660, 0.
Tao, Y., Tang, Y., Yang, Z., Wu, F., Wang, L., Yang, L., Lei, L., Jing, Y., Jiang, X., Jin, H., Bai, Y., Zhang, L., 2020. Exploration of serum exosomal LncRNA TBILA and AGAP2AS1 as promising biomarkers for diagnosis of non-small cell lung cancer. Int. J. Biol. Sci. 16, 471-482. https://doi.org/10.7150/ijbs.39123.

Vaidyanathan, R., Naghibosadat, M., Rauf, S., Korbie, D., Carrascosa, L.G., Shiddiky, M.J. A., Trau, M., 2014. Detecting exosomes specifically: a multiplexed device based on alternating current electrohydrodynamic induced nanoshearing. Anal. Chem. 86, 11125-11132. https://doi.org/10.1021/ac502082b.

Vaidyanathan, R., Soon, R.H., Zhang, P., Jiang, K., Lim, C.T., 2019. Cancer diagnosis: from tumor to liquid biopsy and beyond. Lab Chip 19, 11-34. https://doi.org/ 10.1039/c81c00684a.

Wan, Y., Liu, B., Lei, H., Zhang, B., Wang, Y., Huang, H., Chen, S., Feng, Y., Zhu, L., Gu, Y., Zhang, Q., Ma, H., Zheng, S.Y., 2018. Nanoscale extracellular vesicle-derived DNA is superior to circulating cell-free DNA for mutation detection in early-stage non-small-cell lung cancer. Ann. Oncol. 29, 2379-2383. https://doi.org/10.1093/ annonc/mdy 458 .

Wang, J., Zeng, H., Zhang, H., Han, Y., 2021. The role of exosomal PD-L1 in tumor immunotherapy. Transl. Oncol 14, 101047. https://doi.org/10.1016/j. tranon.2021.101047.

Xu, R., Rai, A., Chen, M., Suwakulsiri, W., Greening, D.W., Simpson, R.J., 2018. Extracellular vesicles in cancer - implications for future improvements in cancer care. Nat. Rev. Clin. Oncol. 15, 617-638. https://doi.org/10.1038/s41571-0180036-9.

Yu, Q., Zhao, Q., Wang, S., Zhao, S., Zhang, S., Yin, Y., Dong, Y., 2020. Development of a lateral flow aptamer assay strip for facile identification of theranostic exosomes isolated from human lung carcinoma cells. Anal. Biochem. 594, 113591. https://doi. org/10.1016/j.ab.2020.113591.

Zhang, Z., Wang, X., Wei, X., Zheng, S.W., Lenhart, B.J., Xu, P., Li, J., Pan, J., Albrecht, H., Liu, C., 2021. Multiplex quantitative detection of SARS-CoV-2 specific IgG and IgM antibodies based on DNA-assisted nanopore sensing. Biosens. Bioelectron. 181, 113134. https://doi.org/10.1016/j.bios.2021.113134.

Zhong, Y., Ding, X., Bian, Y., Wang, J., Zhou, W., Wang, X., Li, P., Shen, Y., Wang, J.J., $\mathrm{Li}$, J., Zhang, C., Wang, C., 2020. Discovery and validation of extracellular vesicleassociated miRNAs as noninvasive detection biomarkers for early-stage non-smallcell lung cancer. Mol. Oncol. 1-14. https://doi.org/10.1002/1878-0261.12889. 